\title{
First-line treatment of malignant glioma with carmustine implants followed by concomitant radiochemotherapy: a multicenter experience
}

\author{
Hans Christoph Bock • Maximilian Josef Anton Puchner • Frauke Lohmann • \\ Michael Schütze • Simone Koll • Ralf Ketter • Ruediger Buchalla • Nikolai Rainov • \\ Sven R. Kantelhardt • Veit Rohde • Alf Giese
}

Received: 9 January 2009/Revised: 29 January 2010 /Accepted: 26 May 2010 /Published online: 13 August 2010

(C) The Author(s) 2010. This article is published with open access at Springerlink.com

\begin{abstract}
Randomized phase III trials have shown significant improvement of survival 1,2 , and 3 years after implantation of 1,3-bis (2-chloroethyl)-1-nitrosourea (BCNU) wafers for patients with newly diagnosed malignant glioma. But these studies and subsequent non-phase III studies have also shown risks associated with local chemotherapy within the central nervous system. The introduction of concomitant radiochemotherapy with temozolomide (TMZ) has later demonstrated a survival benefit in a phase III trial and has become the current treatment standard for newly diagnosed malignant glioma patients. Lately, this has resulted in clinical protocols combining local chemotherapy with BCNU wafers and concomitant radiochemotherapy with $\mathrm{TMZ}$ although this may carry the risk of increased toxicity. We have compiled the treatment experience of seven neurosurgical centers using implantation of carmustine wafers at primary surgery followed by 6 weeks
\end{abstract}

H. C. Bock $\cdot$ S. R. Kantelhardt $\cdot$ V. Rohde $\cdot$ A. Giese

Department of Neurosurgery, Center of Neurological Medicine,

University of Göttingen,

Göttingen, Germany

M. J. A. Puchner · F. Lohmann

Department of Neurosurgery,

Bundesknappschafts-Krankenhaus Recklinghausen,

Recklinghausen, Germany

M. Schütze

Department of Neurosurgery, Rostock University,

Rostock, Germany

\section{S. Koll}

Department of Neurosurgery, Klinikum Fulda,

Saarbrucken, Germany

\section{R. Ketter}

Department of Neurosurgery, Saarland University,

Saarbrucken, Germany of radiation therapy (59-60 Gy) and $75 \mathrm{mg} / \mathrm{m}^{2} /$ day TMZ in patients with newly diagnosed glioblastoma followed by TMZ monochemotherapy. We have retrospectively analyzed the postoperative clinical course, occurrence and severity of adverse events, progression-free interval, and overall survival in 44 patients with newly diagnosed glioblastoma multiforme. All patients received multimodal treatment including tumor resection, BCNU wafer implantation, and concomitant radiochemotherapy. Of 44 patients (mean age $59 \pm 10.8$ years) with glioblastoma who received Gliadel wafer at primary surgery, 28 patients (64\%) had died, 16 patients (36\%) were alive, and 15 patients showed no evidence of clinical or radiographic progression after a median follow-up of 15.6 months. At time of analysis of adverse events in this patient population, the median overall survival was 12.7 months and median progression-free survival was 7.0 months. Surgical, neurolog-

\section{R. Buchalla}

Department of Neurosurgery,

Ev. Luth. Diakonissenanstalt Flensburg,

Flensburg, Germany

\author{
N. Rainov \\ Department of Neurosurgery, Klinikum Augsburg, \\ Augsburg, Germany
}
A. Giese $(\square)$
Department of Neurosurgery,
University of Göttingen,
Robert-Koch-Strasse 40,
37075 Göttingen, Germany
e-mail: alf.giese@med.uni-goettingen.de 
ical, and medical adverse events were analyzed. Twenty-three patients $(52 \%)$ experienced adverse events of any kind including complications that did not require treatment. Nineteen patients (43\%) experienced grade 3 or grade 4 adverse events. Surgical complications included cerebral edema, healing abnormalities, cerebral spinal fluid leakage, meningitis, intracranial abscess, and hydrocephalus. Neurological adverse events included newly diagnosed seizures, alteration of mental status, and new neurological deficits. Medical complications were thromboembolic events (thrombosis, pulmonary embolism) and hematotoxicity. Combination of both treatment strategies, local chemotherapy with BCNU wafer and concomitant radiochemotherapy, appears attractive in aggressive multimodal treatment schedules and may utilize the sensitizing effect of TMZ and carmustine on MGMT and AGT on their respective drug resistance genes. Our data demonstrate that combination of local chemotherapy and concomitant radiochemotherapy carries a significant risk of toxicity that currently appears underestimated. Adverse events observed in this study appear similar to complication rates published in the phase III trials for BCNU wafer implantation followed by radiation therapy alone, but further add the toxicity of concomitant radiochemotherapy with systemic TMZ. Save use of a combined approach will require specific prevention strategies for multimodal treatments.

Keywords Glioma - Local chemotherapy - BCNU .

Carmustine $\cdot$ Gliadel $\cdot$ Temozolomide $\cdot$ Radiochemotherapy

\section{Introduction}

Over the past years, few advances have been made improving progression-free survival (PFS) and overall survival (OAS) of patients with newly diagnosed malignant glioma. 1,3-bis (2Chloroethyl)-1-nitrosourea (BCNU) wafer implantation directly into the resection cavity followed by radiation therapy has shown to significantly improve survival in patients with newly diagnosed malignant glioma [25], and this survival benefit was maintained over the long term [26]. For the subgroup of glioblastoma, the treatment effect in this trail failed to reach statistical significance. However, a meta-analysis combining the results of the randomized phase III trial published by Westphal et al. [25] and an earlier randomized phase III study by Valtonen et al. [24] demonstrated that specifically for the subgroup of glioblastoma treatment with BCNU wafers resulted in a survival increase to 13.1 versus 10.9 months for placebo patients $(p=0.03)$. Representing class II evidence, the results of the two trials lead to Food and Drug Administration (FDA) approval of Gliadel in the treatment of newly diagnosed glioblastoma in March 2003.
Biodegradable BCNU wafers (Gliadel ${ }^{\circledR}$ ) remain the only approved interstitial chemotherapy for treatment of newly diagnosed malignant glioma and recurrent glioblastoma today. More than 20,000 procedures of BCNU wafer implantation into resection cavities have been performed worldwide since the initial release of the product and FDA approval in 1996. Surgically, local intracerebral chemotherapy using BCNU wafers is straightforward, and it is not associated with systemic toxicities $[15,16]$. However, several complications have been associated with implantation of BCNU wafer, including cerebral edema, healing abnormalities, cerebral spinal fluid (CSF) leaks, intracranial infections, seizures, hydrocephalus, and cyst formation. The rates for these adverse events (AE) were well established in two randomized phase III trials that tested $\mathrm{BCNU}$ wafers against placebo wafers $[15,16]$. Since the completion of these studies, systemic chemotherapy for malignant gliomas has made significant progress and has greatly impacted current treatment standards. Stupp et al. demonstrated the efficacy of radiation therapy and concomitant temozolomide in newly diagnosed glioblastomas in a phase III trial [22]. After tumor resection, a 6-week protocol of concomitant radiochemotherapy with temozolomide followed by six cycles of temozolomide monochemotherapy can now be regarded as the treatment standard for patients with newly diagnosed glioblastoma [22]. Generally, this treatment is well tolerated. In the pivotal phase III study of temozolomide in newly diagnosed malignant gliomas, $4.2 \%$ of patients receiving temozolomide plus radiotherapy experienced thromboembolic events. The hematologic toxicities included pancytopenia, thrombocytopenia, and neutropenia. Grades 3 and 4 hematologic toxicity occurred in $16 \%$ of patients receiving chemoradiotherapy with temozolomide [22].

A combination of local BCNU wafer treatment and concomitant radiochemotherapy with temozolomide appears attractive not only because it merges successful treatment strategies for malignant gliomas but it may also take advantage of a sensitizing effect of temozolomide and carmustine on their respective resistance genes MGMT and AGT [18]. Only few data have become available on safety and efficiency of combining local BCNU wafer-based chemotherapy with systemic chemotherapy $[10,14,17]$. Temozolomide has been the most common treatment used with BCNU wafers $[1,4,7,8,10,19]$. Regimen in which temozolomide was administered concomitantly with radiotherapy 2-4 weeks after surgery with BCNU wafer implantation and then followed by temozolomide monochemotherapy has been assessed in small retrospective studies [10, 11, 14, 19]. Further, Asher reported a protocol in which temozolomide was administered for 5 days beginning on postoperative day 4 [1], and a study by Heery et al. used chemoradiotherapy with concomitant temozolomide without additional cycles of a monochemo- 
therapy [8]. These retrospective studies reported no notable increase in the toxicity of BCNU wafer implantation other than the known systemic effects of temozolomide which were mostly hematotoxic events. However, only a small number of patients have been studied, and those studies did not specifically focus on the analysis of adverse events associated with multimodal treatment approaches $[10,13$, $17,20,21]$. Our own experiences in contrast indicate an elevated risk of adverse events than reported in the above studies. In this multicenter toxicity data collection, we therefore have retrospectively analyzed adverse events and the treatment experience of seven neurosurgical centers. Forty-four patients were treated with intraoperative BCNU wafer implantation followed by 6 weeks of daily radiation therapy (59-60 Gy) and concomitant temozolomide (75 mg/ $\mathrm{m}^{2} /$ day) followed by a temozolomide monochemotherapy $\left(150-200 \mathrm{mg} / \mathrm{m}^{2}\right.$ on five consecutive days of a 28-day cycle). All patients had histologically confirmed newly diagnosed glioblastoma. This analysis is focused on complication rates and risk profiles of $\mathrm{BCNU}$ wafer implantation. At time of data analysis, only preliminary survival data were available because of a relatively short follow-up.

\section{Patients and methods}

We have analyzed the clinical course of 44 patients with newly diagnosed glioblastoma WHO grade IV treated at seven neurosurgical institutions. Inclusion criteria for this study were written consent after careful information concerning potential risks and benefits of BCNU wafers, no previous treatment for malignant glioma (operations, chemotherapy, or radiation), and confirmation of a WHO grade IV glioblastoma in the frozen section during operation. Inclusion of the patients for data analysis of adverse events was based on the treatment decision of the individual center. No other formal inclusion criteria applied. All patients underwent initial microsurgical tumor resection (14\% planned partial resection) and implantation of four to eight (mean 7.3 \pm 1.3 ) carmustine-containing wafers (Gliadel Wafers, Archimedes Pharma, Reading, UK) into the resection cavity. Within 4 weeks after surgery, patients were started on oral temozolomide $\left(75 \mathrm{mg} / \mathrm{m}^{2} /\right.$ day) and radiotherapy $1.8 \mathrm{~Gy} /$ day for 6 weeks (total dose ranged from 59 to $60 \mathrm{~Gy}$ ). Within 2-4 weeks after completion of the concomitant radiochemotherapy protocol, patients received a temozolomide monochemotherapy $\left(150-200 \mathrm{mg} / \mathrm{m}^{2}\right)$ for the first 5 days of a 28-day cycle. Time period for data collection started at initial surgery and ranged from August 2005 to August 2008. Potential medical risk factors possibly influencing the clinical course such as diabetes, peripheral vascular disease, or myelosuppression were documented as well as details in surgical techniques for wafer implantation. Clinical data were collected anonymously by datasheet evaluation and analyzed for the study endpoints. These were incidence and grade of adverse events, impact on scheduled oncological treatment, and overall survival and progression-free survival defined by Kaplan-Meyer survival analysis. Adverse events were documented including time of occurrence, severity, necessity of hospitalization, and therapeutic measures taken. Reported adverse events were grouped into surgical, neurological, and medical adverse events and graded according to National Cancer Institute (NCI) guidelines [3].

This study was not undertaken or designed to demonstrate treatment efficacy but to analyze the risks associated with multimodal treatments. However, for survival data analysis at time of data collection including OAS and PFS, Kaplan-Maier analyses were performed; other endpoints were presented by descriptive statistics (WinStat, R. Fitch Software, Bad Krozingen, Germany).

The study cohort included 44 patients (16 females, 28 males) with a mean age of $57 \pm 10.9$ years (range 2874 years) and a mean Karnofsky performance score (KPS) of $81 \pm 15.3 \%$ (range $20-100 \%$ ) at the time of hospital admission (Table 1). Medical risk factors in this patient population included diabetes mellitus in six (14\%) patients, peripheral vascular disease in two $(5 \%)$ patients, and myelosuppression in two (5\%) patients. Surgical risk factors included opening of the ventricular system in 13 patients (30\%) and necessity of a dural patch in 28 patients (64\%). Thirty-seven patients $(84 \%)$ received perioperative prophylactic antibiotic treatment (Table 1).

\section{Results}

At the time of data analysis, 28 patients (64\%) had died and 16 patients $(36 \%)$ were alive with a median follow-up of

Table 1 Baseline demographic characteristics

\begin{tabular}{lccc}
\hline & Mean (SE) & $N(\%)$ & Range \\
\hline Age & $57 \pm 10.9$ & & $28-74$ \\
Karnofsky performance score & $81 \pm 15.3$ & & $20-100$ \\
Medical risk factors & & \\
Diabetes mellitus & $6(14)$ & \\
Peripheral vascular disease & $2(5)$ & \\
Myelosuppression & $2(5)$ & \\
Surgical risk factors & & \\
Opening of the ventricles & & $13(30)$ \\
$\quad$ Dural patch required & & $28(64)$ \\
Planned partial resection & & $6(14)$ \\
Perioperative antibiotics & $37(84)$ \\
\hline
\end{tabular}


15.6 months (Table 2). Postoperative KPS at the time of discharge compared to hospital admission had improved in six patients $(14 \%)$, remained unchanged in 29 patients $(66 \%)$, and decreased in nine patients $(20 \%)$. Ten patients $(23 \%)$ were scheduled for reoperation because of tumor progression during the evaluation period (mean time to reoperation $8.6 \pm 4.8$ months).

\section{Adverse events}

AE of any kind were reported for a total of 23 patients $(52 \%)$ and resulted in therapy delays for concomitant radiochemotherapy in eight patients (18\%) and a deferred start of subsequent temozolomide monochemotherapy in four patients (9\%; Fig. 1). Surgical adverse events included wound healing abnormalities and local wound infections (seven patients, 16\%), CSF leakage (five patients, 11\%), meningitis (three patients, 7\%), intracranial abscess formation (two patients, 5\%), cerebral edema (11 patients, 25\%), and hydrocephalus (three patients, 7\%). Conservative treatment of surgical adverse events included lumbar drainage for CSF leaks, steroids for edema, and i.v. antibiotics for meningitis. Unscheduled readmission to the hospital was required for 19 patients (43\%), and surgical intervention was necessary in seven patients (16\%). Further details for incidence, severity, and treatment of documented adverse events are shown in Table 3.

Neurological AE included new onset seizures (seven patients, 16\%), mental alteration (eight patients, 18\%), and new neurological deficits (five patients, 11\%). Corresponding medical treatment was carried out in all cases.

Prophylactic subcutaneous low molecular weight heparin or i.v. heparin was administered in all patients. Medical adverse events were reported in seven patients (16\%) including thrombosis (four patients, 9\%) and pulmonary embolism (three patients, 11\%). One patient suffered a grade 5 thromboembolic event and died because of pulmonary embolism following deep venous thrombosis during the course of postoperative radiation therapy.

NCI grade 4 leucopenia was observed in one patient $(2 \%)$ and grade 3 thrombopenia in two patients (5\%). One patient with preexisting myelosuppressive disease required readmission for blood transfusion; other patients with low

Table 2 Follow-up and outcome

\begin{tabular}{lcc}
\hline $\begin{array}{l}\text { Median follow-up } \\
\text { (15.6months) }\end{array}$ & $\begin{array}{l}\text { Median OAS } \\
\text { (months) }\end{array}$ & $\begin{array}{l}\text { Median PFS } \\
\text { (months) }\end{array}$ \\
\hline Patients & & \\
Died 28 (64\%) & 10.7 & 6.0 \\
Alive 16 (36\%) & 13.8 & 12.7 \\
All 44 & 12.7 & 7.0 \\
\hline
\end{tabular}

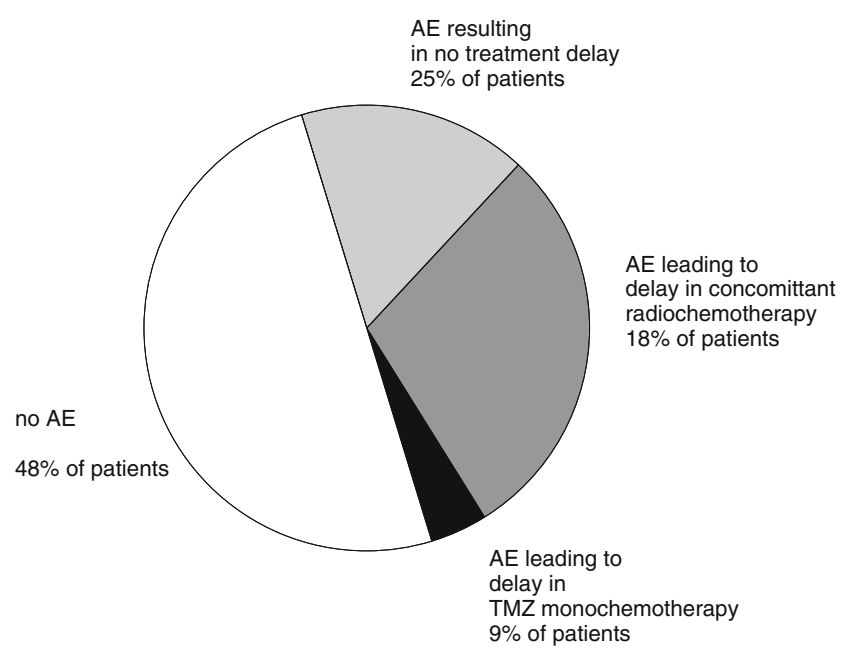

Fig. 1 Impact of $\mathrm{AE}$ on treatment schedule

red or white blood counts did not require transfusion or readmission (Table 3 ).

With respect to time of occurrence of adverse events, it was of interest that complications occurred in 57\% (13 patients) during the postoperative concomitant radiochemotherapy course and in $39 \%$ later on after completion of radiochemotherapy. Only one patient (4\%) was diagnosed with a CSF leak during the immediate postoperative period at day 6 (Fig. 2).

No significant difference in type or frequency of adverse events between the different centers was noted. No significant correlation of preexisting risk factors like age, KPS, diabetes, vascular or myelosuppressive diseases, and the incidence of adverse events was observed. Further, no significant impact of surgical techniques such as dural repair, intraoperative opening of the ventricular system, or perioperative administration of antibiotics was noted.

\section{Survival and progression}

The median OAS in our patient population at time of adverse event analysis was 12.7 months (Fig. 3). However, at the time of data summary, 16 patients $(36 \%)$ were still alive and 15 patients showed no evidence of clinical or radiographic progression. Clinical progression was documented for 30 patients (68\%), and MRI follow-up showed radiographic progression in 18 patients $(41 \%)$. The median follow-up for all patients alive was 15.6 months. The 12-month OS rate was 58\% and median PFS was 7.0 months with a 6-month PFS/12-month PFS rate of $63 \%$ and $35 \%$, respectively.

\section{Discussion}

Recently, we have presented a review of the literature from 1996 to February 2008 on the safety of BCNU wafer in 
Table 3 Adverse events

\begin{tabular}{|c|c|c|c|c|c|}
\hline Type of AE & $\begin{array}{l}\text { Overall } \\
\text { incidence }(\%)^{\mathrm{b}}\end{array}$ & $\begin{array}{l}\text { AE number of } \\
\text { observations }^{c}\end{array}$ & $\begin{array}{l}\text { AE requiring } \\
\text { readmission }[n(\%)]^{\mathrm{d}}\end{array}$ & $\begin{array}{l}\text { AE conservative treatment } \\
\text { alone }[n(\%)]^{\mathrm{d}}\end{array}$ & $\begin{array}{l}\text { AE requiring surgical } \\
\text { intervention }[n(\%)]^{\mathrm{d}}\end{array}$ \\
\hline & 52 & 23 & $19(43)$ & $16(36)$ & $7(16)$ \\
\hline \multicolumn{6}{|l|}{ Surgical/implantation site } \\
\hline Cerebral edema & 25 & 11 & 7 (64) & $11(100)$ & - \\
\hline Healing abnormalities & 16 & 7 & $6(86)$ & $3(43)$ & $4(57)$ \\
\hline CSF leak & 11 & 5 & $5(100)$ & $3(60)$ & $2(40)$ \\
\hline Meningitis & 7 & 3 & $2(67)$ & $3(100)$ & - \\
\hline Intracranial abscess & 5 & 2 & $2(100)$ & - & $2(100)$ \\
\hline Hydrocephalus & 7 & 3 & $2(67)$ & $2(67)$ & $1(33)$ \\
\hline \multicolumn{6}{|l|}{ Neurological } \\
\hline New seizures & 16 & 7 & $7(100)$ & $7(100)$ & - \\
\hline Alteration of mental status & 18 & 8 & $6(75)$ & $8(100)$ & - \\
\hline New neurological deficits & 11 & 5 & $2(40)$ & $5(100)$ & - \\
\hline \multicolumn{6}{|l|}{ Medical } \\
\hline Deep vein thrombosis & 9 & 4 & $3(75)$ & $4(100)$ & - \\
\hline Pulmonary embolism & 7 & 3 & $3(100)$ & $3(100)$ & - \\
\hline Leucopenia $^{\mathrm{a}}$ & 2 & 1 & - & $1(2)$ & - \\
\hline Thrombocytopenia $^{\mathrm{a}}$ & 5 & 2 & $1(50)$ & $2(100)$ & - \\
\hline Anemia $^{\mathrm{a}}$ & - & - & - & - & - \\
\hline
\end{tabular}

${ }^{\mathrm{a}} \mathrm{NCI}$ grades 3 and 4

${ }^{\mathrm{b}} n=44$ patients for the denominator

${ }^{\mathrm{c}}$ In 44 patients

${ }^{\mathrm{d}} \mathrm{AE}$ number of observations for the denominator

newly diagnosed and recurrent malignant glioma patients. Three pivotal clinical phase III trials and 26 non-phase III studies were analyzed. The treatment experience for implantation of BCNU wafers in patients with newly diagnosed malignant glioma and patients with recurrent disease were surveyed, and the overall incidence of adverse events was summarized [19]. BCNU wafer group $\mathrm{AE}$ rates from the pivotal phase III trials for newly diagnosed glioblastoma ranged from $4 \%$ to $23 \%$ for cerebral edema, $4 \%$ to $9 \%$ for intracranial hypertension, $14 \%$ to $16 \%$ for healing abnormalities, $5 \%$ for CSF leaks, $4 \%$ to $5 \%$ for intracranial infection, $19 \%$ to $33 \%$ for seizures, $10 \%$ for deep vein thrombosis, and $8 \%$ for pulmonary embolus. For newly diagnosed disease, statistically significant differences between BCNU wafer treatment and the placebo groups were observed only for

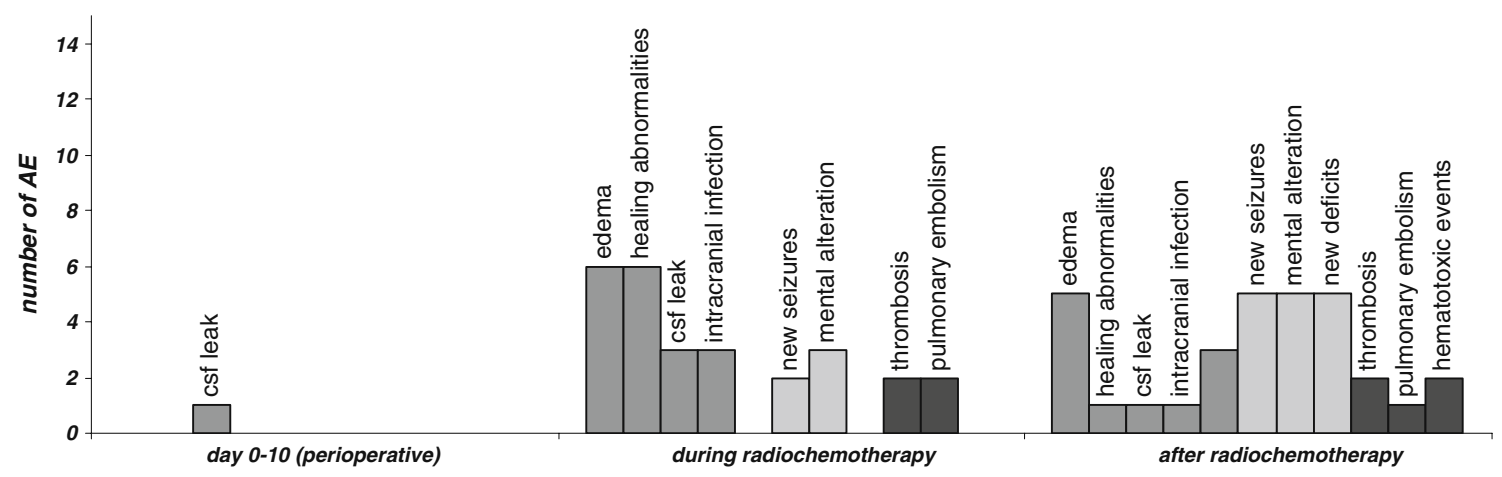

Fig. 2 Time course of AE occurrence in 44 patients 
Fig. 3 Survival and progression in 44 glioblastoma patients treated with carmustine wafers and concomitant radiochemotherapy

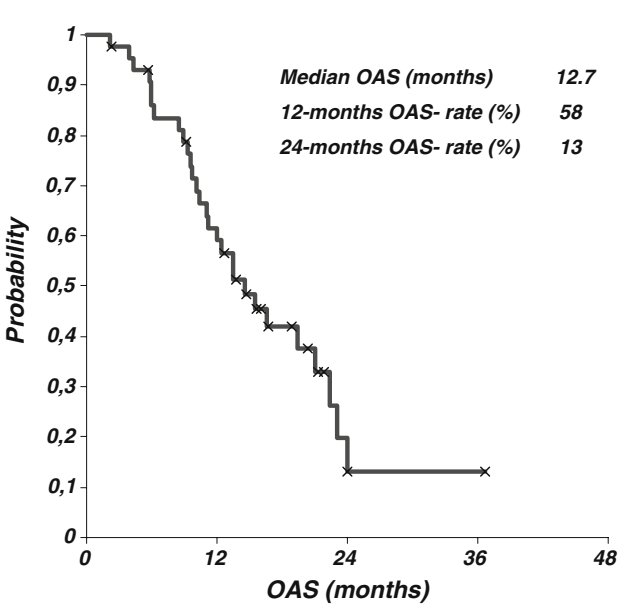

intracranial hypertension which occurred at $>6$ months following implantation in nine of the 11 patients in $\mathrm{BCNU}$ wafer-treated patients. This was attributed to tumor recurrence and subsequent mass effect. CSF leak was more common in the BCNU wafer group than in the placebo wafer group, but did not reach statistical significance [25]. However, for both BCNU wafer and placebo wafer treatment, wound healing abnormalities and cerebral edema seemed higher than expected in a general population of brain tumor patients undergoing craniotomy. This may not be unexpected, as animal studies have reported an inflammatory response to blank polyanhydride copolymers [23].

In the phase III trial enrolling patients with recurrent malignant gliomas, the overall incidence of serious intracranial infection was more common with BCNU wafer treatment (four out of $110,4 \%$ ) compared with placebo (one out of $112,1 \%$ ), although the difference was not statistically significant [2]. However, there was a significant difference in the incidence of wound healing abnormalities (14\% for the BCNU wafer group and 5\% for the placebo wafer group; $p<0.05)$. While similar proportions of patients had seizures postoperatively, there was a difference in the proportion of patients experiencing new or worsened postoperative seizures early postoperatively. There were no differences in $\mathrm{AE}$ rates between the three phase III studies and 26 non-phase III studies. For the non-phase III studies, the overall incidence of $\mathrm{AE}$ was low, ranging from $0.2 \%$ for intracranial hypertension to $9.6 \%$ for healing abnormalities. Healing abnormalities, intracranial infection, and seizures were the most consistently reported AE $[5,14$, 26]. Rates of healing abnormalities appeared higher in recurrent than in newly diagnosed disease [19].

While data from the Gliadel phase III studies suggested that BCNU wafer is generally well tolerated and effective, its use has evolved in the context of new treatment standards for malignant gliomas. Currently, most patients receiving $\mathrm{BCNU}$ wafer will also receive radiation and systemic chemotherapy, as part of multimodal treatment strategies. This has led to combination of BCNU wafer with brachytherapy $[4,19,27]$, radiosurgery $[1,7,10,12,19$, 20], and systemic therapies including irinotecan, lomustine, procarbazine, carboplatin, interferon, and temozolomide [9, 11]. However, multimodal treatment strategies carry the potential of additive toxicity and therefore have to be evaluated extremely carefully. Temozolomide is the most recent agent that has proven efficacy in the treatment of malignant gliomas. Temozolomide was shown to significantly prolong survival in patients with newly diagnosed malignant gliomas and is generally well tolerated, with hematologic toxicity being the most notable safety issue [22]. The combination of local BCNU wafer treatment and systemic temozolomide has not been assessed in phase III trials, but recently, some non-phase III studies have investigated the efficacy and safety of BCNU wafer and temozolomide within multimodal treatment strategies $[1,7$, 10, 19]. There were no unexpected AE reported in the BCNU wafer and temozolomide studies. Compared to known AE associated with BCNU wafer treatment, the added hematological toxicity at a median rate of $14.8 \%$ (7.1-53.3\%) was comparable to grade 3 or 4 hematologic toxic events reported in the phase III study for temozolomide alone [22]. Combining local and systemic treatment strategies is tempting. However, elevated complication rates may lead to delays in oncological treatment and may even result in early termination of treatment plans. The risk/ benefit ratio for these intense multimodal treatments has therefore to be assessed very carefully. In contrast to the few other studies investigating intraoperative BCNU wafer treatment followed by postoperative radiation therapy and concomitant temozolomide and subsequent temozolomide monochemotherapy, our multicenter study focused on the retrospective analysis of $\mathrm{AE}$ specifically and was not designed nor powered to demonstrate treatment efficacy. Inclusion of patients with newly diagnosed glioblastoma for analysis of treatment-related adverse events was based on the treatment decision of the individual center. No other 
formal inclusion criteria such as age, neurological performance score, or feasibility of a gross total resection applied. Therefore, this patient population is likely to represent a negative selection bias in terms of prognosis and treatment risk compared to phase III trials or other studies (compare material and methods section). However, this patient population is likely to reflect a cohort requiring treatment in every day neuro-oncology practice. In this patient population, our analysis suggests a higher rate of $\mathrm{AE}$ compared to the data published for the BCNU wafer phase III trials $[15,16]$. We found an elevated incidence of intracranial infections and CSF leaks (Table 4) compared to a recently published study [19] that reported a comparatively low incidence of adverse events for BCNU wafer treatment and concurrent temozolomide. Only three of 21 patients experienced toxic side effects. A recent report on a similar phase II study [10] reported an overall high rate of adverse events (20 of 35 patients), but did not specifically report on wound healing abnormalities. One intracranial abscess was reported in the later analysis. However, there was an unexpected rate of thromboembolic events (deep vein thrombosis in five and pulmonary embolism in four of 35 patients), respectively. A study designed to specifically analyze adverse events is likely to find an elevated rate of such events. The non-phase III studies cited above focused on treatment efficacy and did not present clear criteria for adverse event reporting. In this study, 23 patients (52\%) experienced adverse events of any kind which included complications that did not require specific treatment. Nineteen patients (43\%) experienced grade 3 or grade 4 adverse events including hematotoxic events. This compares to $16 \%$ grade 3 or grade 4 events for concomitant radiochemotherapy [22] added to the adverse events reported in the phase three trials for local BCNU chemotherapy followed by radiation. These trails did not report how many patients experienced grade 3 or grade 4 adverse events, but rather presented rates for specific events, for example, $16 \%$ wound healing, $5 \%$ intracranial infection, and $33 \%$ seizures [25]. Therefore, it is difficult to estimate the additional risk of the multimodal approach studied here. In our study for eight patients $(18 \%)$, AE resulted in the delay of radiochemotherapy, and for four patients (9\%), AE lead to delay of further oncological treatment. It is likely that surgical strategies or preexisting medical conditions impact on the incidence of adverse events. Surprisingly, this was not observed in our study. Frequency and type of adverse events were independent of age, preoperative clinical condition, surgical strategy, or the institution at which patients were treated. Interestingly, all adverse events registered in this study occurred after scheduled discharge from hospital and during the first 2 to 3 months after BCNU wafer implantation. In 19 patients (43\%), readmission in a neurosurgical center was required, and specific neurosurgical intervention was necessary in seven patients (16\%). Because most adverse events occurred during concomitant radiochemotherapy or through the first cycles of temozolomide monochemotherapy, the relatively short follow-up of this study seems to be sufficient to evaluate the risk of $\mathrm{AE}$ under this treatment regimen. Our study suggests that the incidence of adverse events associated with BCNU wafer treatment in combination with concurrent radiation and temozolomide chemotherapy is currently underestimated. We have therefore recently conducted a comprehensive review of the literature on individual adverse events associated with carmustine wafer implantation reported in the literature and the proposed avoidance

Table 4 Incidence of AE compared

\begin{tabular}{|c|c|c|c|}
\hline Type of AE & $\begin{array}{l}\text { Overall incidence }(\%) \\
\text { Newly diagnosed GBM BCNU } \\
\text { wafer+radiation }+ \text { TMZ chemotherapy } \\
\text { ( } n=44 \text { patients) }\end{array}$ & $\begin{array}{l}\text { Overall incidence }(\%) \\
\text { Newly diagnosed GBM pivotal } \\
\text { phase III trial BCNU wafer } \\
\text { reatment group }(n=120 \text { patients })^{\text {a }}\end{array}$ & $\begin{array}{l}\text { Overall incidence }(\%) \\
\text { Selected non-phase III clinical } \\
\text { studies of BCNU wafer treatment } \\
\text { in newly diagnosed and recurrent } \\
\text { malignant gliomas }(n=887 \text { patients })^{\mathrm{b}}\end{array}$ \\
\hline Healing abnormalities & 16 & 16 & 9.6 \\
\hline CSF leak & 11 & 5 & 2.1 \\
\hline Intracranial infection & 9 & 5 & 2.4 \\
\hline \multicolumn{4}{|l|}{ Thromboembolic events } \\
\hline Deep vein thrombosis & 9 & 10 & 1.6 \\
\hline Pulmonary embolism & 7 & 8 & 1.0 \\
\hline \multicolumn{4}{|l|}{ Hematotoxic events } \\
\hline NCI grades 3 and 4 & 5 & 0 & Not analyzed \\
\hline
\end{tabular}

\footnotetext{
${ }^{a}$ Westphal et al. [25]

${ }^{\mathrm{b}}$ Sabel and Giese [19]

GBM glioblastoma multiforme
} 
strategies [6]. Based on the reported incidence and the time course of adverse events, we have proposed a formal protocol for patient selection, surgical techniques, and follow-up schedules for patients scheduled for carmustine wafer implantation at our institution. Following introduction and strict adherence to this risk management protocol, specifically implantation site-related complications dropped significantly [6]. Our study further demonstrates that several adverse events occur late after initial surgery and wafer implantation (compare Fig. 2). Most adverse events, including wound healing abnormalities and intracranial infections, occurred after patients had been discharged from neurosurgical units and manifested at a time when patients were not under direct care of their surgeons. Therefore, the neuro-oncological follow-up schedule was now modified to account for late wound healing complications [6].

\section{Conclusion}

The efficacy of BCNU wafer treatment followed by radiation chemotherapy within multimodal treatment strategies to day remains unproven and therefore has to be considered an experimental treatment strategy. The safety data provided here suggest that $\mathrm{BCNU}$ wafer treatment followed by radiation chemotherapy requires further validation of risk management strategies. The use of BCNU wafer with radiation and concurrent temozolomide followed by temozolomide monochemotherapy does not appear to result in new types of AE. However, clinicians should be aware of the potential risks associated with $\mathrm{BCNU}$ wafer.

\section{Conflict of Interest None.}

Open Access This article is distributed under the terms of the Creative Commons Attribution Noncommercial License which permits any noncommercial use, distribution, and reproduction in any medium, provided the original author(s) and source are credited.

\section{References}

1. Asher A (2007) Prospective analysis of temozolomide as adjuvant to Gliadel and radiation in newly diagnosed malignant glioma. Abstract presented at the Annual Meeting of the American Association of Neurological Surgeons, Washington, DC 2007

2. Brem H, Piantadosi S, Burger PC, Walker M, Selker R, Vick NA, Black K, Sisti M, Brem S, Mohr G et al (1995) Placebo-controlled trial of safety and efficacy of intraoperative controlled delivery by biodegradable polymers of chemotherapy for recurrent gliomas. The Polymer-brain Tumor Treatment Group. Lancet 345:10081012

3. Cancer Therapy Evaluation Program (2006) Common terminology criteria for adverse events, version 3.0, DCTD, NCI, NIH,
DHHS, March 31, 2003 (http://ctep.cancer.gov). Publish Date: August 9

4. Darakchiev BJ, Albright RE, Breneman JC, Warnick RE (2008) Safety and efficacy of permanent iodine-125 seed implants and carmustine wafers in patients with recurrent glioblastoma multiforme. J Neurosurg 108:236-242

5. Fung LK, Ewend MG, Sills A, Sipos EP, Thompson R, Watts M, Colvin OM, Brem H, Saltzman WM (1998) Pharmacokinetics of interstitial delivery of carmustine, 4-hydroperoxycyclophosphamide, and paclitaxel from a biodegradable polymer implant in the monkey brain. Cancer Res 58:672-684

6. Giese A, Bock HC, Kantelhardt SR, Rohde V (2010): Risk Management in the Treatment of Malignant Gliomas with BCNU Wafer Implants. Jan 8. doi:10.1055/s-0029-1242775

7. Gururangan S, Cokgor L, Rich JN, Edwards S, Affronti ML, Quinn JA, Herndon JE 2nd, Provenzale JM, McLendon RE, Tourt-Uhlig S, Sampson JH, Stafford-Fox V, Zaknoen S, Early M, Friedman AH, Friedman HS (2001) Phase I study of Gliadel wafers plus temozolomide in adults with recurrent supratentorial high-grade gliomas. Neuro Oncol 3:246-250

8. Heery CR, Desjardins A, Quinn JA, Rich JN, Gururangan S, Vredenburgh JJ et al (2006) Acute toxicity analysis of patients receiving surgery, Gliadel wafer implantation, and postoperative daily temozolomide with radiation therapy for primary high-grade glioma. Journal Clinical Oncology, ASCO Annual Meeting Proceedings (Post-Meeting Edition), vol 24(18S), p 11504

9. LaRocca R, Glisson S, Hargis J, Petruska D, Villanueva W, Morassutti D et al (2006) High-grade glioma treated with surgery; carmustine wafer; postoperative radiation; and procarbazine, lomustine, and vincristine chemotherapy. Neurosurg Q 15 (3): $167-171$

10. LaRocca RV, Hodes J, Villanueva WG, Vitaz TW, Morassutti DJ, Doyle MJ, et al (2006) A phase II study of radiation with concomitant and then sequential temozolomide (TMZ) in patients with newly diagnosed supratentorial high-grade malignant glioma who have undergone surgery with carmustine (BCNU) wafer insertion: Abstract presented at the Annual Meeting of the Society of Neuro-oncology, Orlando, FL (Abstract)

11. Lawson DH, Phuphanich S, McKenzie E, Greiner K, Shires K, Olson J (2006) Phase I analysis of systemic interferon alpha utilized with gliadel in adults with recurrent glioblastoma multiforme (GBM). Journal of Clinical Oncology, 2006 ASCO Annual Meeting Proceedings Part I, Vol 24, No 18S (June 20 Supplement), p 11508

12. Limentani SA, Asher A, Heafner M, Kim JW, Fraser R (2005) A phase I trial of surgery, Gliadel wafer implantation, and immediate postoperative carboplatin in combination with radiation therapy for primary anaplastic astrocytoma or glioblastoma multiforme. $\mathrm{J}$ Neurooncol 72:241-244

13. McGirt MJ, Than KD, Weingart JD, Chaichana KL, Attenello FJ, Olivi A, Laterra J, Kleinberg LR, Grossman SA, Brem H, Quiñones-Hinojosa A (2009) Gliadel (BCNU) wafer plus concomitant temozolomide therapy after primary resection of glioblastoma multiforme. J Neurosurg 110(3):583-588

14. McGovern PC, Lautenbach E, Brennan PJ, Lustig RA, Fishman NO (2003) Risk factors for postcraniotomy surgical site infection after 1, 3-bis (2-chloroethyl)-1-nitrosourea (Gliadel) wafer placement. Clin Infect Dis 36:759-765

15. MGI Pharma (2006) Gliadel ${ }^{\circledR}$ Wafer [prescribing information] MGI PHARMA, Bloomington

16. MGI Pharma (2006) Gliadel ${ }^{\circledR}$ Wafer [summary of product characteristics] MGI PHARMA, West Sussex, UK

17. Pan E, Mitchell SB, Tsai JS (2008) A retrospective study of the safety of BCNU wafers with concurrent temozolomide and radiotherapy and adjuvant temozolomide for newly diagnosed glioblastoma patients. J Neurooncol 88:353-357 
18. Plowman J, Waud WR, Koutsoukos AD, Rubinstein LV, Moore TD, Grever MR (1994) Preclinical antitumor activity of temozolomide in mice: efficacy against human brain tumor xenografts and synergism with 1, 3-bis(2-chloroethyl)-1-nitrosourea. Cancer Res 54:3793-3799

19. Sabel M, Giese A (2008) Safety profile of carmustine wafers in malignant glioma: a review of controlled trials and a decade of clinical experience. Curr Med Res Opin 24(11):3239-3257

20. Sampath P, Weaver CE, Alderson L, Duncan JA, Stopa E, Fountas KN (2005) Consecutive use of brachytherapy (Gliasite) and intratumoral chemotherapy (Gliadel) for recurrent glioblastoma: a phase I/II study with independent clinical review. Poster and abstract presented at the Annual Meeting of the Congress of Neurological Surgeons, Boston, MA, (Abstract)

21. Smith KA, Ashby L, Gonzolas F (2006) Gross total resection (GTR) with Gliadel ${ }^{\circledR}$ Wafer implantation followed by postoperative gamma knife radiosurgery and adjuvant fractionated radiotherapy for newly diagnosed GBM: phase I/II study of safety and efficacy in 30 consecutive patients. Abstract presented at the Annual Meeting of the Congress of Neurological Surgeons, Chicago, IL (Abstract)

22. Stupp R, Mason WP, van den Bent MJ, Weller M, Fisher B, Taphoorn MJ et al (2005) Radiotherapy plus concomitant and adjuvant temozolomide for glioblastoma. N Engl J Med 352:987996

23. Tamargo RJ, Epstein JI, Reinhard CS, Chasin M, Brem H (1989) Brain biocompatibility of a biodegradable, controlled-release polymer in rats. J Biomed Mater Res 23:253-266

24. Valtonen S, Timonen U, Toivanen P, Kalimo H, Kivipelto L, Heiskanen O, Unsgaard G, Kuurne T (1997) Interstitial chemotherapy with carmustine-loaded polymers for high grade gliomas: A randomized double-blind study. Neurosurg 41:44-49

25. Westphal M, Hilt DC, Bortey E, Delavault P, Olivares R, Warnke PC et al (2003) A phase 3 trial of local chemotherapy with biodegradable carmustine (BCNU) wafers (Gliadel wafers) in patients with primary malignant glioma. Neuro Oncol 5:79-88

26. Westphal M, Ram Z, Riddle V, Hilt D, Bortey E (2006) Gliadel wafer in initial surgery for malignant glioma: long-term follow-up of a multicenter controlled trial. Acta Neurochir (Wien) 148:269275, discussion 275

27. Zamorano L, Li Q, Levin K, Barger G (2005) Brachytherapy with I-125 permanent implant combined with sequential Gliadel in the treatment of patients with GBM. Abstract presented at the Annual Meeting of the Congress of Neurological Surgeons, Boston, MA (Abstract)

\section{Comments}

\section{Miguel A. Arraez, Malaga, Spain}

The article written by Bock and colleagues is focusing on the treatment of malignant gliomas by means of surgery, BCNU wafers in the tumoral bed, radiotherapy, and temozolomide. The addition of BCNU wafers as local therapy has been proven useful in malignant gliomas, although with untoward side effects. The Stupp scheme (radiation therapy and concomitant temozolomide/6 weeks radiochemotherapy followed by six cycles of temozolomide monochemotherapy) has also shown its potential to prolong survival in these patients. The current management of malignant gliomas varies according patients and centers, but it is important to notice that nowadays many patients undergo resection and receive BCNU wafers and after discharge from hospital, radiotherapy plus temozolomide is administered. In this paper, Bock et al. do state that the main aim of this study is not the survival analysis but the adverse effects of such a combination of treatments. This is a very important endpoint for the analysis. The results of Bock and colleagues show a surprisingly high rate of complications and adverse effects, more than probably suspected, when the above-mentioned combination of treatments is used. It calls also the attention of the high number of thromboembolic events in this subset of patients. It is not known whether this series of patients did receive or not prophylaxis with heparin.

The results of this study suggest that a further selection of patients to follow this scheme should be regarded, as the risk/benefits ratio could not be the same for all patients. According to this consideration, some stratification could be made in the future taking into account survival and adverse events for obvious purposes of model prediction before undertaking the combination of surgery, BCNU wafers, radiation, and temozolomide.

\section{Oliver Heese, Hamburg, Germany}

The currently available standard for the treatment of glioblastoma consists of gross total resection followed by concomitant radiation therapy/chemotherapy with temozolomide followed by cyclic temozolomide for 6 months resulting in an overall survival of 14 to 15 months with an acceptable side effect spectrum. In theory, intensifying this regimen by adding a local chemotherapy may lead to a prolonged survival, in particular since time from surgery to the beginning of radiation therapy is without any further treatment so far. In this nicely written manuscript, a multicenter approach was taken to gain information regarding the number and severity of adverse events of a "first-line treatment of malignant glioma with carmustine implants followed by concomitant radiochemotherapy". In 44 patients, the adverse events were collected retrospectively. Treatment efficacy data were not the intention of the study due to the retrospective and unrandomized trial design, but a median overall survival of 12.7 months and a median progression-free survival of 7.0 months were seen.

Regarding the number of adverse events, $52 \%$ of patients experienced adverse events of any kind including complications not needing further treatment, and in $43 \%$ of patients' adverse events, grades 3 and 4 were seen. The authors summarize that a combination of local chemotherapy and concomitant radiochemotherapy carries a significant risk of toxicity that may have been underestimated currently.

In conclusion, these data definitely lead to caution when planning new therapy protocols for malignant glioma treatment. It seems that $1+1$ is not always 2 and combining different therapy regimens, in theory from a pathophysiological perspective working synergistically, may lead to an increased and unacceptable side effects spectrum. Taking into account that all of these therapies are only performed in a palliative setting and so far the overall survival for glioblastoma multiforme (GBM) is still only approximately 14 month, severe side effects leading to hospitalization and profound loss of quality of life are unacceptable for patients and their families. For further study designs, focusing only on endpoints like overall survival and progression-free survival may not be adequate, and adding quality of life assessments and other psycho-oncological criteria seems to be mandatory in order to do the best for GBM patients. 